\title{
Spin-lattice effects in selected antiferromagnetic materials (Review Article)
}

\author{
S. Zherlitsyn, S. Yasin, and J. Wosnitza \\ Hochfeld-Magnetlabor Dresden (HLD), Helmholtz-Zentrum Dresden-Rossendorf, Dresden D-01314, Germany \\ E-mail: s.zherlitsyn@hzdr.de
}

\section{A.A. Zvyagin}

B. Verkin Institute for Low Temperature Physics and Engineering of the National Academy of Sciences of Ukraine 47 Lenin Ave., Kharkov 61103, Ukraine

\author{
A.V. Andreev \\ Institute of Physics ASCR, Na Slovance 2, 18221 Prague 8, The Czech Republic \\ V. Tsurkan \\ Universität Augsburg, Augsburg 86159, Germany \\ Institute of Applied Physics, Academy of Sciences of Moldova, Chisinau MD-2028, R. Moldova
}

Received August 29, 2013

\begin{abstract}
Spin-lattice effects play an important role in many magnetic materials. In this short review, we give some examples of such effects studied in low-dimensional, frustrated as well as uranium-based antiferromagnets. Utilizing ultrasound measurements at low temperatures and high magnetic fields provides valuable information on the spin-strain interactions. Specifically phase transformations and critical phenomena in magnetic systems with strong spin-lattice interactions are fruitful grounds for sound-velocity and sound-attenuation measurements.
\end{abstract}

PACS: 72.55.+s Magnetoacoustic effects;

75.45.+j Macroscopic quantum phenomena in magnetic systems;

62.65.+k Acoustical properties of solids;

75.50.Ee Antiferromagnetics.

Keywords: low-dimensional spin systems, frustrated chromium spinels, spin-strain interaction, uranium-based compounds.

\section{Contents}

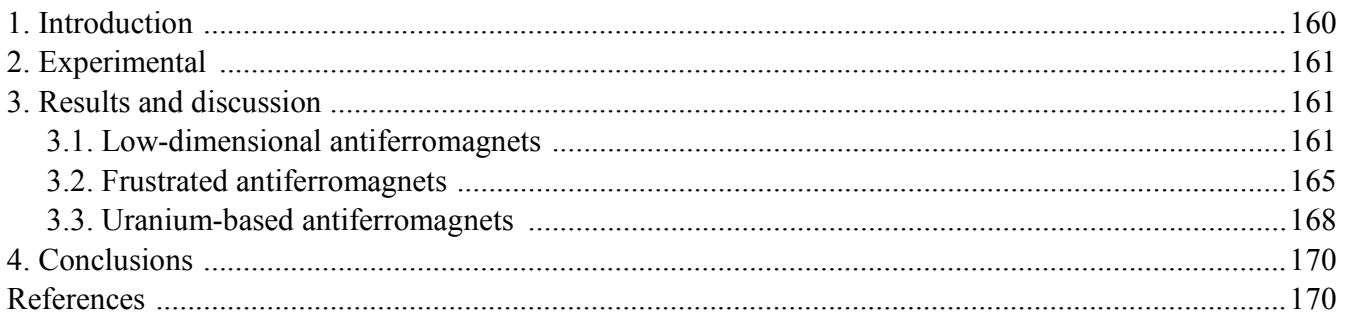

\section{Introduction}

Interest in spin-strain interactions and their role in magnetism has grown considerably in recent years. Magnetic systems with magnetic ions at certain crystallographic positions are usually described by a model Hamiltonian con- taining various contributions (e.g., crystal electric field (CEF) potential, magnetic exchange, magnetic anisotropy, dipolar interaction, Dzyaloshinsky-Moriya (DM) interaction, the RKKY interaction in case of conducting materials and so on). Generally, the magnetic interactions depend on the magnetic-ion neighborhood as well as ion-ion distances. 
Therefore, any displacement in the system leads to a renormalization of these interactions defining the coupling to the lattice degrees of freedom. This coupling results in certain spin-lattice effects both static (shifts of ion positions) and dynamic (coupling to phonons). Various aspects of magneto-elastic interactions have been addressed in a number of monographs [1-5] and reviews [6-9].

Here we discuss spin-lattice effects originating mainly from the exchange-striction mechanism caused by the renormalization of inter-ionic magnetic interactions due to spin-phonon coupling. We do not consider here single-iontype effects as well as effects associated with coupling to spin waves or domain-wall effects. We will give a few examples of spin-lattice phenomena studied in selected low-dimensional systems, frustrated magnets, and uranium-based materials.

\section{Experimental}

Ultrasound investigations are a powerful experimental technique which is well established as an important tool for materials research [1]. There are as well a number of approaches to measure the sound velocity and sound attenuation in magnetic materials to study spin-phonon interactions. Most of the results described here have been obtained using a well-proven experimental method based on a phase-sensitive detection technique [10]. By means of this technique the relative change of the sound velocity, $\Delta v / v$, and sound attenuation, $\Delta \alpha$, is measured with high accuracy as a function of external parameters. The sound velocity is related to the elastic modulus via $c=\rho v^{2}$, where $\rho$ is the mass density. Figure 1 shows a simplified scheme of the ultrasound setup (for more details see, for instance, Ref. 10).

The relative change of the sound velocity, is proportional to the frequency change, $\Delta \omega / \omega$, phase change, $\Delta \varphi / \varphi$, and sample length change $\Delta L / L: \Delta v / v=\Delta \omega / \omega-$ $-\Delta \varphi / \varphi+\Delta L / L$. The last contribution is usually negligible. From the giving expression one can immediately see two possibilities for sound-velocity measurements: 1) to determine the frequency change by keeping the phase constant, or 2) to extract the phase change at constant frequency. In the first case, the measurements are more precise with a typical resolution of the order of $10^{-6}$. In the later case, the resolution is typically reduced by one order of magnitude. But here the advantage is that, in contrast to the first option data acquisition is relatively fast and the method is well suited in combination with pulsed magnetic fields.

Technically, the two detection schemes utilize the same ultrasound setup (see Fig. 1) but differ mainly by activating a phase modulation (to keep the phase constant) and a feedback loop to match the frequency with the soundvelocity change. The sound attenuation can be easily obtained from the change in the amplitude of the transmitted ultrasound signal [10].

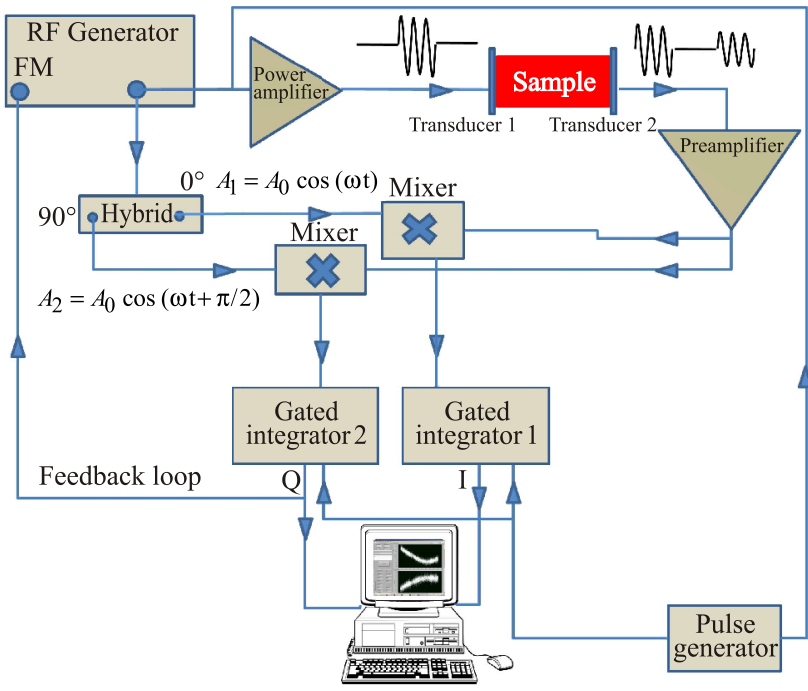

Fig. 1. (Color online) Simplified setup used for the soundvelocity and sound-attenuation measurements, based on a standard pulse-echo method and phase-sensitive detection technique. An RF pulse excites high-frequency mechanical oscillations in the piezoelectric transducer. The ultrasound wave propagates through the sample. It is detected with another transducer on the opposite side of the sample. The received RF signal is amplified and mixed with two phase-shifted reference signals having the same frequency. After suppressing higher harmonics with lowpass filters (not shown) and integrating the resulting signals with gated box-car averagers there are two output dc signals ( $I$ and $Q$ ), from which the sound velocity and attenuation can be extracted. For more details see Ref. 10.

Typically, samples demand some preparation for ultrasound experiments. Single crystals have to be oriented and polished for attaching piezoelectric transducers. Commercial resonance $\mathrm{LiNbO}_{3}$ and wide-frequency-band polyvinylidene fluoride (PVDF) film transducers are used to excite and to detect ultrasonic waves at frequency of 30-150 MHz. Two types of pulsed-field magnets have been used. One magnet capable of reaching about $70 \mathrm{~T}$ in a bore of $24 \mathrm{~mm}$ having a pulse rise time of $33 \mathrm{~ms}$ and a total pulse duration of about $150 \mathrm{~ms}$. Further, a $90 \mathrm{~T}$ dual-coil magnet with $16 \mathrm{~mm}$ bore has been used. For details about the pulsed magnets see Refs. 11, 12. Static magnetic fields up to $20 \mathrm{~T}$ were produced by a commercial superconducting magnet combined with a variable temperature insert and a ${ }^{3} \mathrm{He}$ cryostat.

\section{Results and discussion}

\subsection{Low-dimensional antiferromagnets}

In this section, we summarize our magneto-acoustic investigations at the quantum $S=1$ spin-chain magnet dichloro-tetrakis thiourea-nickel(II), $\mathrm{NiCl}_{2}-4 \mathrm{SC}\left(\mathrm{NH}_{2}\right)_{2}$, known as DTN [13-15], and at the quasi-two-dimensional $S=1 / 2$ 
antiferromagnet $\mathrm{Cs}_{2} \mathrm{CuCl}_{4}\left(T_{N} \approx 0.6 \mathrm{~K}\right)[16,17]$. Both materials have been discussed in the context of Bose-Einstein condensation (BEC) of magnons [18-22].

DTN has a body-centered tetragonal crystal structure (space group I4), with two formula units per unit cell. Inelastic neutron diffraction [18] and electron spin resonance [23] studies reveal that DTN is a quasi-1D spin system $\left(J_{c}=\right.$ $=2.2 \mathrm{~K}, J_{a, b}=0.18 \mathrm{~K}$ ) with a strong easy-plane single-ion anisotropy $(D=8.9 \mathrm{~K})$, which opens a gap in the lowlying spin excitations. When an external magnetic field, $H$, is applied along the spin chains, the gap closes and an $X Y$-like antiferromagnetic (AFM) ordering occurs at $H_{c}=2.1 \mathrm{~T}$; increasing the field, the spins are fully polarized at $H_{S}=$ $=12.6 \mathrm{~T}$. At zero temperature, these critical fields correspond to two quantum critical points (QCPs). The maximum temperature for the AF phase is $T_{N}^{\max } \approx 1.2 \mathrm{~K}$ at $H \approx$ $\approx 5.7 \mathrm{~T}$ (see inset of Fig. 3) [24].

The DTN single crystal used for the ultrasound measurement had a size of about $2 \times 2 \times 4.1 \mathrm{~mm}$. For our experiments, we propagated sound waves along the [001] direction with polarization along the same direction, corresponding to the acoustic $c_{33}$ mode. By propagating sound along the spin chains, the distance between the magnetic ions is affected, and the intra-chain exchange interaction is modulated (exchange-striction coupling).

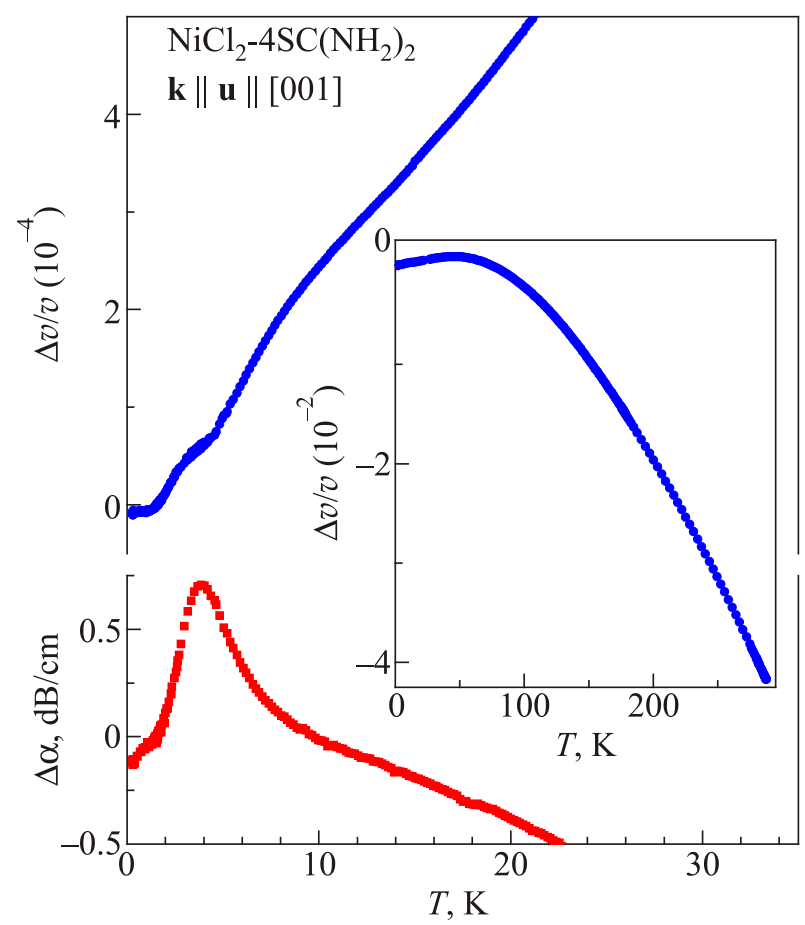

Fig. 2. (Color online) Sound velocity, $\Delta v / v$, of the longitudinal $c_{33}$ mode in DTN, as a function of temperature in zero magnetic field (upper panel and inset). The sound velocity has a maximum at $\sim 45 \mathrm{~K}$. The measurements were performed at a frequency of $78 \mathrm{MHz}$. Lower panel shows a change of attenuation, $\Delta \alpha$, as a function of temperature, measured simultaneously with the sound velocity. Here $\mathbf{k}$ and $\mathbf{u}$ are the wave vector and polarization of the ultrasonic wave, respectively.
Figure 2 shows the relative change of the sound velocity, $\Delta v / v$, and sound attenuation, $\Delta \alpha$, of the $c_{33}$ mode versus temperature in zero magnetic field. The sound velocity grows steadily from room temperature down to about $45 \mathrm{~K}$, where it reaches a maximum, and then decreases again below $45 \mathrm{~K}$. This softening below $45 \mathrm{~K}$ cannot be attributed to exchange-striction coupling, because the magnetic exchange-energy scale is much smaller. In this temperature range, single-ion effects most probably renormalize $v$. Weaker anomalies can be seen for $T<10 \mathrm{~K}$, in the same range where the attenuation shows a pronounced maximum at about $4 \mathrm{~K}$ [15]. This can be ascribed to $1 \mathrm{D}$ spin fluctuations.

Figure 3 shows the temperature dependence of $\Delta v / v$ and $\Delta \alpha$ for the $c_{33}$ mode in DTN obtained at $6 \mathrm{~T}$ applied along the [001] direction. At this field, $T_{N}^{\max } \approx 1.2 \mathrm{~K}$. Clear anomalies are seen both in the sound velocity and sound attenuation at $1.2 \mathrm{~K}$; both quantities show a jump-like decrease when entering the AFM state. This is caused by the suppression of 1D spin fluctuations in the ordered state.

Figure 4 shows magnetic-field dependences of $\Delta v / v$ and $\Delta \alpha$ for the $c_{33}$ mode for temperatures between 0.3 and $1.2 \mathrm{~K}$. The phase transitions corresponding to $H_{c}$ and $H_{s}$ result in sharp anomalies in $\Delta v / v$ and in $\Delta \alpha$ for temperatures below $T_{N}^{\max }$. The anomalies become more pronounced and narrower with decreasing temperature. This reflects a strong spin-lattice coupling within the AFM phase, with relative changes in $\Delta v / v$ as large as $0.5 \%$, as the spins are canted by the magnetic field. The $H$ dependence of $\Delta v / v$ in the ordered phase resembles $c$-axis magnetostriction data [25], but the magnetostriction is too small $\left(\sim 10^{-4}\right)$ to explain our data. The sound velocity for temperatures close to $T_{N}^{\max }$ shows the same qualitative $H$ dependence as below $T_{N}^{\max }$, but with significantly suppressed and smoothed anomalies. The attenuation peaks are comparable to those at low temperatures, which implies

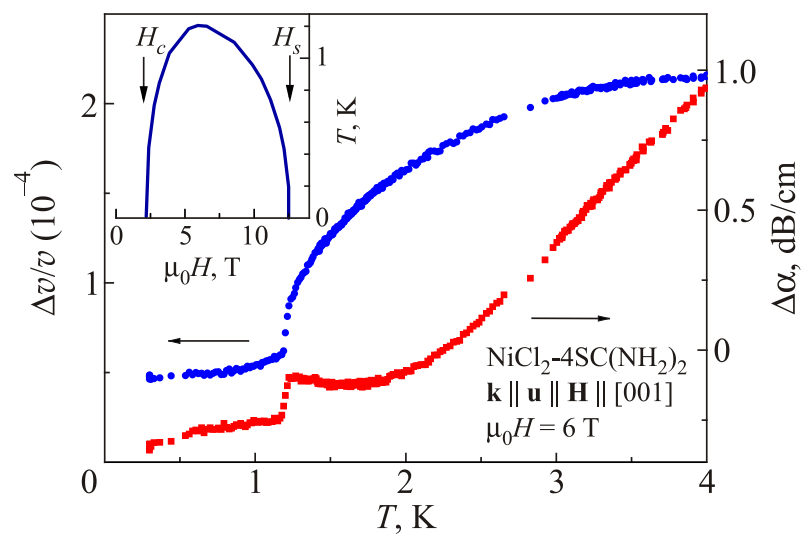

Fig. 3. (Color online) Temperature dependence of the sound velocity, $\Delta v / v$, and sound attenuation, $\Delta \alpha$, of the acoustic $c_{33}$ mode measured in an applied magnetic field of $6 \mathrm{~T}$ [15]. The inset shows the $H-T$ phase diagram of DTN. 


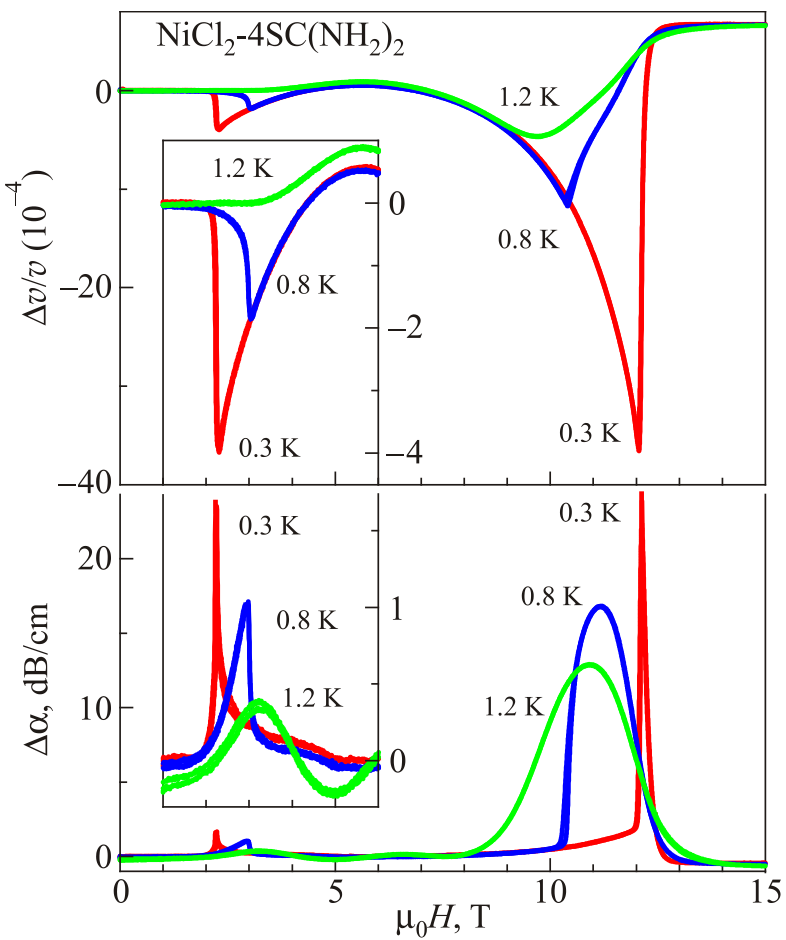

Fig. 4. (Color online) Relative change of the sound velocity, $\Delta v / v$, (top) and attenuation, $\Delta \alpha$, (bottom) of the acoustic $c_{33}$ mode at $78 \mathrm{MHz}$ as a function of magnetic field applied along the [001] direction. The data were taken at constant temperature. The insets show the data in enlarged scales close to $H_{c}$.

that the energy of the sound wave is dissipated close to the critical regions at $H_{c}$ and $H_{s}$, even at temperatures far from the actual quantum critical points.

Using exchange-striction approach, a model based on an effective free-fermion theory for a 1D $S=1$ spin chain has been developed, which gives a good qualitative description of the sound velocity and attenuation outside the AFM phase [13].

In magnetic materials the dominant contribution to spin-lattice interactions mostly arises from the exchangestriction coupling. According to Ref. 26, the relative renormalization of the longitudinal sound velocity can be written as

$$
\frac{\Delta v}{v}=-\frac{A_{1}+A_{2}}{\left(N \omega_{\mathbf{k}}\right)^{2}},
$$

where

$$
\begin{gathered}
A_{1}=2\left|G_{0}^{z}(\mathbf{k})\right|^{2}\left\langle S_{0}^{z}\right\rangle^{2} \chi_{0}^{z}+T \sum_{\mathbf{q}} \sum_{\alpha=x, y, z}\left|G_{\mathbf{q}}^{\alpha}(\mathbf{k})\right|^{2}\left(\chi_{\mathbf{q}}^{\alpha}\right)^{2}, \\
A_{2}=H_{0}^{z}(\mathbf{k})\left\langle S_{0}^{z}\right\rangle^{2}+\frac{T}{2} \sum_{\mathbf{q}} \sum_{\alpha=x, y, z} H_{\mathbf{q}}^{\alpha}(\mathbf{k}) \chi_{\mathbf{q}}^{\alpha} .
\end{gathered}
$$

Here, $N$ is the number of spins in the system, $\omega_{\mathbf{k}}=v k$ is the low- $k$ dispersion relation with sound velocity $v$ in the absence of spin-phonon interactions, $\left\langle S_{0}^{z}\right\rangle$ is the average magnetization along the direction of the magnetic field, $\chi_{\mathbf{q}}^{x, y, z}$ are non-uniform magnetic susceptibilities, and the subscript 0 corresponds to $q=0$. The renormalization is proportional to the spin-phonon coupling constants $G_{\mathbf{q}}^{\alpha}$ and $H_{\mathbf{q}}^{\alpha}$ which are used as fit parameters. For spin systems with antiferromagnetic interactions, such as DTN, the main contribution to the summation over $\mathbf{q}$ in Eq. (2) comes from terms with $q=\pi$. The temperature and magnetic-field dependence of the uniform and non-uniform magnetic susceptibility of a quasi-1D spin system has been calculated in mean-field approximation in the framework of the effective free-fermion model [13,27].

Following Ref. 26, the attenuation coefficient for DTN can be written as

$$
\begin{aligned}
\Delta \alpha & \left.\equiv \Delta \alpha_{k}\right)=\frac{1}{N v}\left[2\left|G_{0}^{z}(\mathbf{k})\right|^{2}\left\langle S_{0}^{z}\right\rangle^{2} \chi_{0}^{z} \frac{\gamma_{0}^{z}}{\left(\gamma_{0}^{z}\right)^{2}+\omega_{\mathbf{k}}^{2}}+\right. \\
& \left.+T \sum_{\mathbf{q}} \sum_{\alpha=x, y, z}\left|G_{\mathbf{q}}^{\alpha}(\mathbf{k})\right|^{2}\left(\chi_{\mathbf{q}}^{\alpha}\right)^{2} \frac{2 \gamma_{\mathbf{q}}^{\alpha}}{\left(2 \gamma_{\mathbf{q}}^{\alpha}\right)^{2}+\omega_{\mathbf{k}}^{2}}\right],
\end{aligned}
$$

where $\gamma_{\mathbf{q}}^{\alpha}$ are the relaxation rates, which can be approximated by $\gamma_{\mathbf{q}}^{\alpha}=B / T \chi_{\mathbf{q}}^{\alpha}$, where $B$ is a material-dependent constant (see Ref. 26). Here an approximation is used, in which the relaxation rates do not depend on the direction and amplitude of the wave vector. The results of the calculations for the sound velocity and sound attenuation in DTN are presented in Figs. 5 and 6, respectively. The model reproduces the main features of the experimentally observed dependences, such as the pronounced minimum in $\Delta v / v$ at $H_{s}$, the almost field-independent behavior at $H>H_{s}$ and $H<H_{c}$, the larger value of $\Delta v / v$ for $H>H_{s}$ as compared to $H<H_{c}$, and the maximum (with $\Delta v / v>0$ ) in the interval between $H_{c}$ and $H_{s}$. With increasing $T$ the

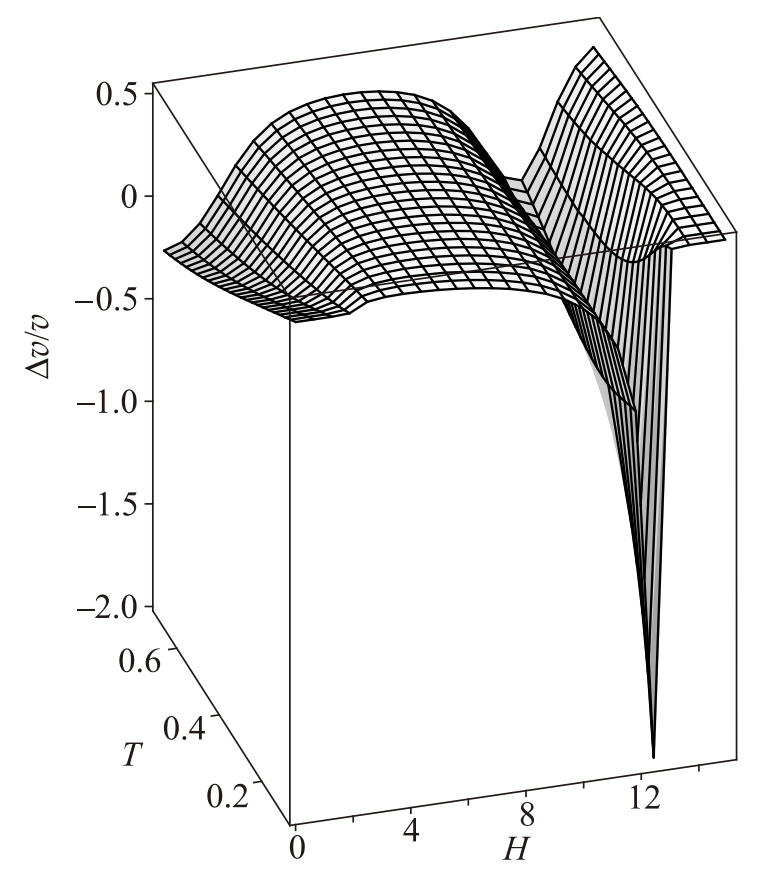

Fig. 5. Relative change of the velocity of the longitudinal sound versus $H$ and $T$, calculated in the framework of the free-fermion model (see Ref. 13 for details). 


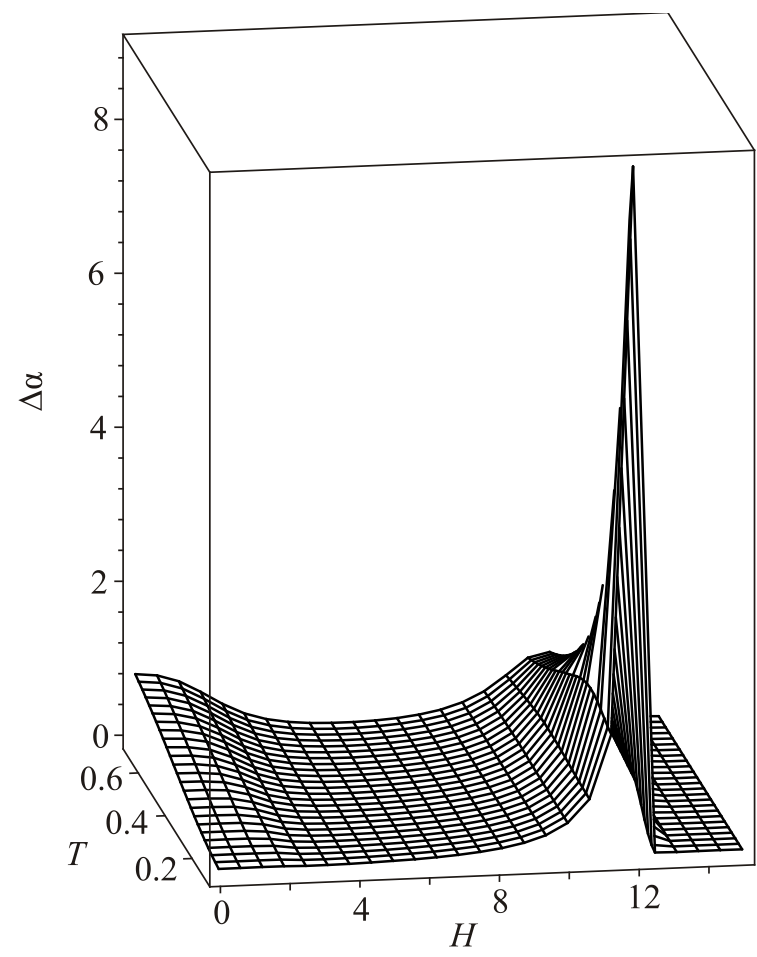

Fig. 6. Attenuation of the longitudinal sound versus $H$ and $T$, calculated in the framework of the free-fermion model (see Ref. 13 for details).

anomalies near the critical fields become weaker, the same way as it was observed in experiment (cf. Figs. 3 and 4). The calculated attenuation (Fig. 6) reproduces as well the main features observed in the experimental data: an abrupt increase of the sound attenuation near the saturation field, and decreasing anomalies with increasing $T$. All these findings evidence the important role the fermionic magnetic excitations play in the vicinity of the QCPs in DTN.

Now, we proceed with reviewing the results obtained in the quasi-two-dimensional $S=1 / 2$ antiferromagnet $\mathrm{Cs}_{2} \mathrm{CuCl}_{4}$. This compound has attracted a lot of interest as a candidate for a spin-liquid material in the triangular $S=1 / 2$ antiferromagnetic lattice. This quantum phase is strictly defined only at zero temperature, but its effect can also be realized at higher temperatures [28]. For $\mathrm{Cs}_{2} \mathrm{CuCl}_{4}$, neutronscattering experiments $[29,30]$ indicate the existence of this phase. According to the neutron-scattering, specific-heat [19,31], susceptibility, and magnetization [31] measurements, a long-range incommensurate antiferromagnetic order appears below $T_{N}=0.6 \mathrm{~K}$. For fields applied along different crystallographic axes a number of magnetic phases have been observed [31]. Above $\mu_{0} H(T=0) \sim 8.5 \mathrm{~T}$, a saturated state is reached. In the temperature range $T_{N}<T \lesssim 2.6 \mathrm{~K}$, the compound shows spin-liquid hallmarks. Above $2.6 \mathrm{~K}$, the susceptibility obeys a Curie-Weiss law [31].

$\mathrm{Cs}_{2} \mathrm{CuCl}_{4}$ has an orthorhombic Pnma crystal structure. The in-plane $b c$ interactions, $J=4.34 \mathrm{~K}$ along $b$ and $J^{\prime}=0.34 J$ along zig-zag bonds, are dominant with respect to the interplanar interaction, $J^{\prime \prime}=0.045 J$. These values

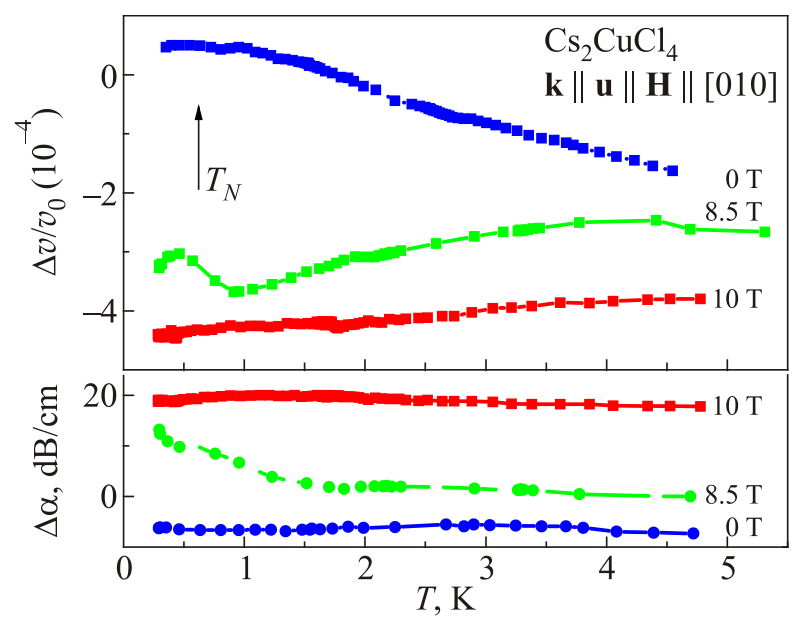

Fig. 7. (Color online) Temperature dependence of the relative change of the velocity and attenuation of the acoustic $c_{22}$ mode in $\mathrm{Cs}_{2} \mathrm{CuCl}_{4}$ in different magnetic fields probed by a longitudinal ultrasound wave at $42 \mathrm{MHz}$. The experimental geometry is $\mathbf{H}\|\mathbf{k}\| \mathbf{u} \|[010]$.

were estimated from the measured magnon dispersion in the saturated phase [32]. Measurements of the relative change of the sound velocity and sound attenuation for the longitudinal acoustic $c_{22}$ mode $(\mathbf{k}\|\mathbf{u}\|[010])$ in this material are reported in Refs. 16, 17. In this geometry, the longitudinal acoustic wave modulates the largest exchange interaction $J$. The magnetic field was applied as well along the [010] direction. Results for acoustic $c_{22}$ and $c_{33}$ mode as well as a theoretical analysis based on the spinwave expansion have been reported in Ref. 33 .

At zero magnetic field, the sound velocity grows linearly with decreasing temperature and does not reveal any anomalies down to low temperatures (see Fig. 7). In an applied field of $8.5 \mathrm{~T}$, the temperature dependence of the sound velocity reveals a lattice softening below about $4.5 \mathrm{~K}$ and develops a non-monotonous structure around the Néel temperature of $0.62 \mathrm{~K}$. This structure disappears in data taken at $10 \mathrm{~T}$. For the sound velocity an increase towards the lowest temperatures can be seen at 8.5 T. In Fig. 8, we present field

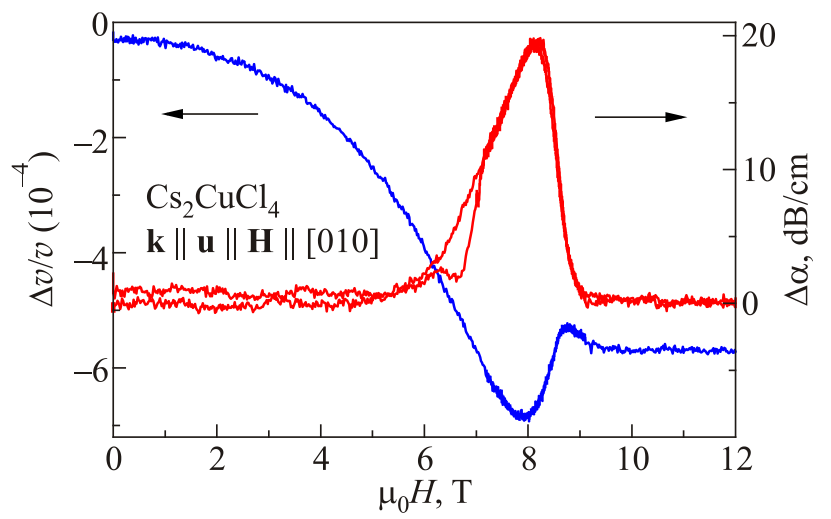

Fig. 8. (Color online) Field dependence of the relative change of the velocity and attenuation of the acoustic $c_{22}$ mode measured with an ultrasound frequency of $42 \mathrm{MHz}$ at $T=300 \mathrm{mK}$. Both up and down sweeps are shown for the sound attenuation. 
dependences of the acoustic properties measured at $300 \mathrm{mK}$. The sound velocity shows a softening with increasing field and becomes constant in the fully polarized phase above $H_{S}$ demonstrating a strong dip anomaly around $H_{s}$. The sound attenuation exhibits a strong peak at about $H_{S}$ for temperatures below $1.2 \mathrm{~K}$ [16].

Assuming that in $\mathrm{Cs}_{2} \mathrm{CuCl}_{4}$ the spatial dependence of the magnetic anisotropy is weaker than the spatial dependence of the exchange integrals one can use the Eqs. (1)-(3) to calculate the sound velocity and sound attenuation above the ordered state (for more details see Ref. 16). Similar to DTN, in $\mathrm{Cs}_{2} \mathrm{CuCl}_{4}$ the main contribution to the summation over $\mathbf{q}$ in Eq. (2) comes from terms with $q^{x, y, z}=\pi$. In contrast to DTN, the calculations were done in the framework of the hard-core boson theory for low-energy spin excitations for the spin subsystem of $\mathrm{Cs}_{2} \mathrm{CuCl}_{4}$ [16]. Again, the ultrasound attenuation in $\mathrm{Cs}_{2} \mathrm{CuCl}_{4}$ can be calculated with the help of Eq. (3) using approximations in which the relaxation rates do not depend on the direction and amplitude of the wave vector. The calculations reproduce the main features observed in the experimental data: the pronounced minimum in the field dependence of the sound velocity, the peak in the attenuation at about $H_{s}$, and the almost field-independent behavior of the acoustic properties at $H \gtrsim H_{s}$ [16]. All these findings demonstrate the important role which the magnetic excitations play in the vicinity of $T_{N}(H)$ and support the conclusions of previous investigations in $\mathrm{Cs}_{2} \mathrm{CuCl}_{4}$ [29,30], which interpret the behavior of the magnetic properties above the Néel temperature and below $H_{s}$ as the manifestation of a spinliquid state.

\subsection{Frustrated antiferromagnets}

In this section, we address spin-lattice effects observed in selected antiferromagnetic chromium spinels. A characteristic structural feature of the chromium spinels is the pyrochlore lattice consisting of corner-sharing tetrahedra with $\mathrm{Cr}^{3+}$ ions $(S=3 / 2)$ sitting on the corners. The structural peculiarities provide the background for both, geometric and bond frustrations caused by competing magnetic interactions. This leads to unusual ground states in these materials. Oxide spinels are geometrically frustrated having direct antiferromagnetic exchange of the order of a few hundred Kelvin, whereas the sulfide and selenide spinels are bond frustrated due to a competition between the direct AFM exchange and indirect $90^{\circ} \mathrm{Cr}-\mathrm{X}-\mathrm{Cr}$ ferromagnetic exchange interactions with the ground state still being antiferromagnetic (X being S or Se) [34].

Here, we discuss ultrasound results obtained on $\mathrm{CdCr}_{2} \mathrm{O}_{4}$, $\mathrm{CoCr}_{2} \mathrm{O}_{4}, \mathrm{ZnCr}_{2} \mathrm{~S}_{4}$, and $\mathrm{ZnCr}_{2} \mathrm{Se}_{4}$ [35-39]. At room temperature these materials possess a cubic structure with space group $F d \overline{3} m$. In external magnetic fields the oxide spinels show magnetization plateaus with a spin configuration having three spins up and one spin down for each tetrahedron, whereas the magnetization in $\mathrm{ZnCr}_{2} \mathrm{~S}_{4}$ and $\mathrm{ZnCr}_{2} \mathrm{Se}_{4}$ increases rather smoothly with field (see, for instance, Fig. 13, lower panel) [38,39]. Strong spin-phonon coupling leads to a rich variety of spin-lattice effects including magnetostructural phase transitions. Since the orbital moments are quenched in these compounds, spin-orbit coupling is negligible and magnetic anisotropy is not important. We have studied the acoustic modes $c_{11}$ and $c_{L}=1 / 3\left(c_{11}+2 c_{12}+4 c_{44}\right)$ [35-39], with $c_{i j}$ being the elastic constants for a cubic crystal. $c_{L}$ corresponds to a longitudinal acoustic mode propagating along the [111] direction.

First we discuss the temperature dependences of the sound velocity in the chromium spinels. As an example, results obtained at various applied magnetic fields for the sound velocity of the $c_{L}$ mode in $\mathrm{CoCr}_{2} \mathrm{O}_{4}$ are shown in Fig. 9. In this material, $\mathrm{Co}^{2+}$ ions with electronic configuration $3 d^{7}$ and spin $S=3 / 2$ occupy tetrahedral sites, whereas $\mathrm{Cr}^{3+}\left(3 d^{3}, S=3 / 2\right)$ ions are situated at octahedral positions. A collinear ferrimagnetic spin order sets in below $T_{C}=95 \mathrm{~K}$, followed by a transition into an incommensurate (IC) long-range conical-spiral state at $T_{S}=27 \mathrm{~K}$ [41-45]. The spin configuration below $T_{l}=14 \mathrm{~K}$ is commensurate. A further remarkable property of $\mathrm{CoCr}_{2} \mathrm{O}_{4}$ is its multiferroicity. A spontaneous dielectric polarization appears in the IC-spiral state below $T_{S}$ [46], indicating a low-symmetry phase. This multiferroicity in $\mathrm{CoCr}_{2} \mathrm{O}_{4}$ was assigned to the inverse Dzyaloshinskii-Moriya interaction based on the fact that the observed modulations of the spins and the lattice have the same wave vector [47].

Distinct anomalies with significant changes in the sound velocity are seen in this spinel compound at the onset of long-range incommensurate-spiral-spin order at $T_{S}=27 \mathrm{~K}$ and at the transition from the incommensurate to the commensurate state at $T_{l}$. At the transition to the collinear ferrimagnetic state at $T_{C}$ only a small anomaly is observed at zero magnetic field. Additionally, between $T_{C}$ and the

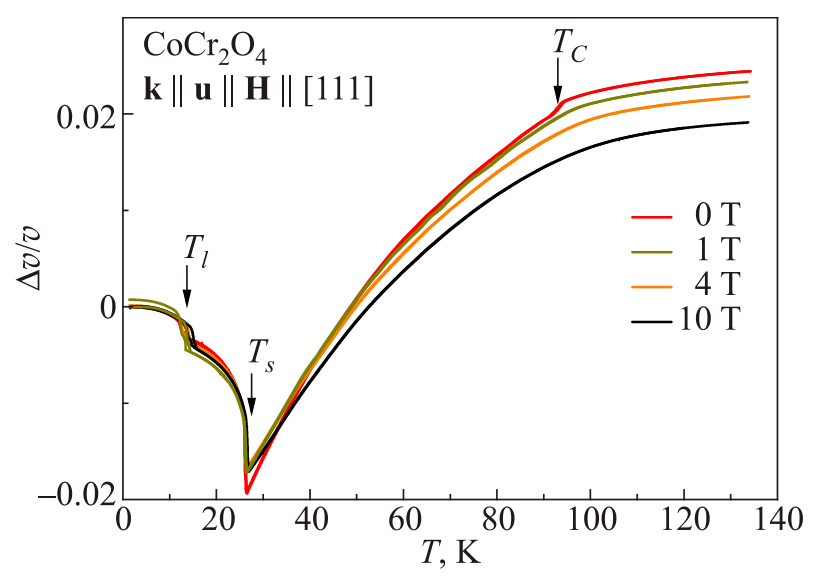

Fig. 9. (Color online) Temperature dependence of the sound velocity, $\Delta v / v$, for $\mathrm{CoCr}_{2} \mathrm{O}_{4}$ measured in different static magnetic fields for ultrasound waves propagating along the [111] axis. The arrows mark the magnetic phase transitions $T_{C}, T_{s}$, and $T_{l}$ observed at zero magnetic field. 
transition into the IC spiral spin state at $T_{S}$, a considerable lattice softening of $\Delta v / v$ of about $4 \%$ appears in $\mathrm{CoCr}_{2} \mathrm{O}_{4}$.

In the high-temperature cubic phase, the sound velocity decreases toward lower temperatures, contrary to what is observed in most solids. A similar strong softening in the paramagnetic phase is observed for the related frustrated spinel compounds $\mathrm{CdCr}_{2} \mathrm{O}_{4}$ [35,36], $\mathrm{ZnCr}_{2} \mathrm{~S}_{4}$, and $\mathrm{ZnCr}_{2} \mathrm{Se}_{4}$ [38,39]. Similar behavior was previously reported for $\mathrm{ZnCr}_{2} \mathrm{O}_{4}$ [40]. The strong softening of the measured elastic constants in the paramagnetic phase can be ascribed to the coupling between the spin correlations and phonons and is successfully described by models similar to that mentioned in the previous section. By fitting the temperature dependences of the sound velocity the spin-lattice coupling constant can be extracted [35].

We now concentrate on high-field ultrasound results obtained on $\mathrm{CoCr}_{2} \mathrm{O}_{4}, \mathrm{ZnCr}_{2} \mathrm{~S}_{4}$, and $\mathrm{CdCr}_{2} \mathrm{O}_{4}$ [35-37]. Figure 10 shows $\Delta v / v$ of $\mathrm{CoCr}_{2} \mathrm{O}_{4}$ as function of applied pulsed magnetic field at different temperatures. At $4.4 \mathrm{~K}$, the sound velocity increases monotonously with magnetic field in a fully reversible manner. This reflects the field dependence of the sound velocity in the spiral spin configuration, which is stable up to $62 \mathrm{~T}$. In contrast, at $6.7 \mathrm{~K}$ a step-like increase in the sound velocity and a kink-like sharp maximum in the attenuation (not shown) are found at $55 \mathrm{~T}$, signaling the transition into a new high-field phase with significantly enhanced stiffness. The sound velocity in this state is close to the velocity in the vicinity of $T_{C}$ (see Fig. 9). This hints at a structural similarity of the new high-field phase and the high-symmetry phase above $T_{C}$. Astonishingly, upon reducing the applied field, the ground state is not recovered at the same magnetic-field values $H_{t}$, but the high-field phase remains metastable down to a lower transition field and seems to persist even to zero field at $6.7 \mathrm{~K}$. The situation changes at higher temperatures. At $16.5 \mathrm{~K}$, the metastable state exists between approximately 40 and $60 \mathrm{~T}$, whereas at $30 \mathrm{~K}$ no metastable state is seen

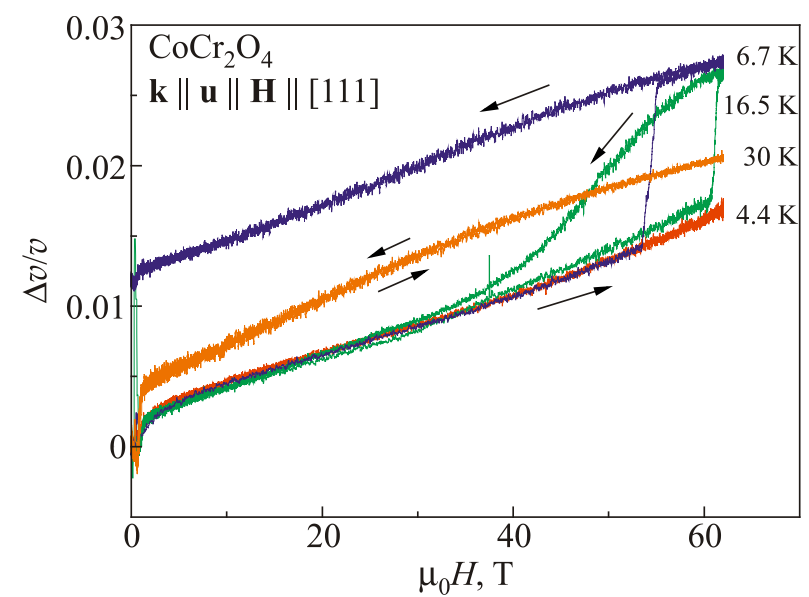

Fig. 10. (Color online) Relative change of the sound velocity, $\Delta v / v$, of the acoustic $c_{L}$ mode in $\mathrm{CoCr}_{2} \mathrm{O}_{4}$ at different temperatures versus magnetic field applied along the [111] direction. Data for up and down field sweeps are shown. The arrows indicate the field-sweep directions.

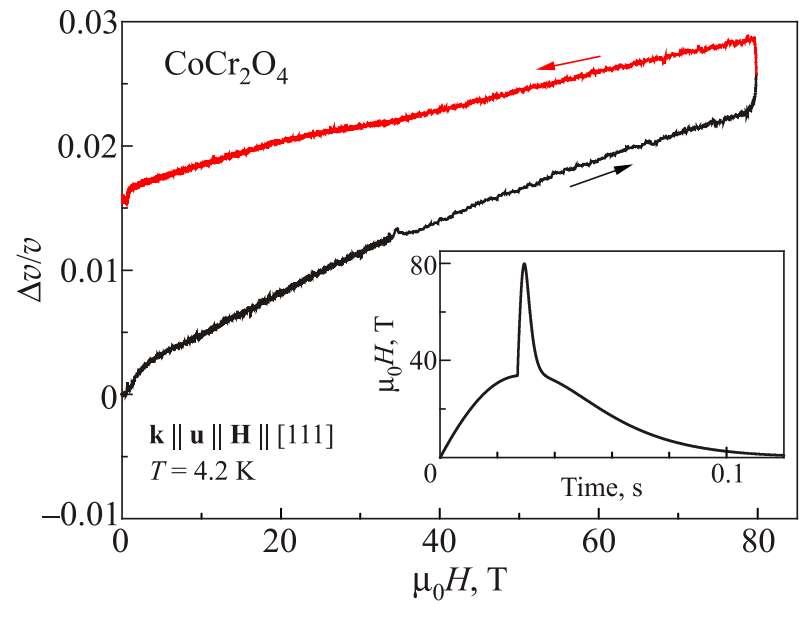

Fig. 11. (Color online) Relative change of the sound velocity, $\Delta v / v$, of the acoustic $c_{L}$ mode in $\mathrm{CoCr}_{2} \mathrm{O}_{4}$ at $4.2 \mathrm{~K}$ measured in a pulsed field up to $80 \mathrm{~T}$ applied along the [111] direction. Data for up and down field sweeps are shown. The arrows indicate the field-sweep directions. The inset shows the time dependence of the magnetic field, obtained using a 9.5 MJ dual-coil pulsed magnet (see Refs. 11, 12 for details).

up to $63 \mathrm{~T}$. It is worth noting that the magnetization does not demonstrate any anomalies in this temperature and magnetic-field range [37].

Using a dual-coil 9.5 MJ pulsed magnet we measured the sound velocity change at $4.2 \mathrm{~K}$ up to $80 \mathrm{~T}$ (Fig. 11). The peak field is very close to the phase transition into the high-field phase with the metastable phase stretching from $80 \mathrm{~T}$ down to $0 \mathrm{~T}$ (compare with fully reversible behavior at $4.4 \mathrm{~K}$ up to $62 \mathrm{~T}$ shown in Fig. 10). The whole $H-T$ phase diagram of $\mathrm{CoCr}_{2} \mathrm{O}_{4}$ extracted from the ultrasound experiments is presented in Fig. 12.

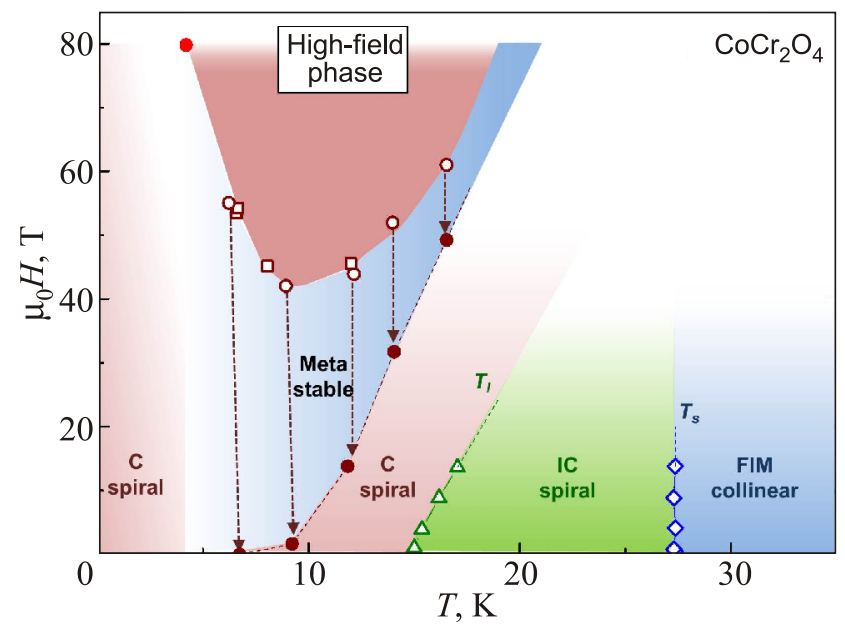

Fig. 12. (Color online) Low-temperature $H-T$ phase diagram of $\mathrm{CoCr}_{2} \mathrm{O}_{4}$ extracted from the ultrasound measurements. The metastable phase appears when passing the high-field phase boundary towards lower fields. The metastable phase leads to a hysteresis in the acoustic properties (Figs. 10 and 11). The red point at about $80 \mathrm{~T}$ is obtained from the data shown in Fig. 11. For more details see Ref. 37 . 
Our data evidence that in the high-field (high-symmetry) phase only the longitudinal component of the magnetization is ordered, while the long-range transverse spiral order is suppressed [37]. Concomitantly, effects of the inverse DM interaction are canceled and the structure is released into presumably a cubic symmetry. The high-field state remains metastable on lowering the field until the transverse magnetization is large enough to reestablish the spiral state. The leading contribution of spin-lattice coupling and the origin of multiferroicity is assigned to arise from the inverse DM interaction and not from the conventional symmetric exchange-striction coupling.

Very fascinating acoustic properties have been observed at high (pulsed) magnetic fields in $\mathrm{ZnCr}_{2} \mathrm{~S}_{4}$. In this bond-frustrated material, below the antiferromagnetic transition at $T_{N 1} \approx 14 \mathrm{~K}$, the sound velocity reveals a sequence of steps followed by plateaus as a function of magnetic field evidencing a succession of crystallographic structures with constant stiffness (Fig. 13) [38]. The magnetization, on the other hand increases smoothly with field up to saturation without any plateaus (Fig. 13, lower panel). This contrasts the magnetization data measured in geometrically frustrated chromium-oxide spinels [48,49]. Using the ultrasound results, the $H-T$ phase diagram of $\mathrm{ZnCr}_{2} \mathrm{~S}_{4}$ was constructed (Fig. 14) [38].

A very different behavior is found in $\mathrm{CdCr}_{2} \mathrm{O}_{4}[35,36]$. In this material, the Curie-Weiss temperature, $\Theta_{C W}=-70 \mathrm{~K}$, is an order of magnitude larger than the Néel temperature indicating a high level of magnetic frustration [48]. The

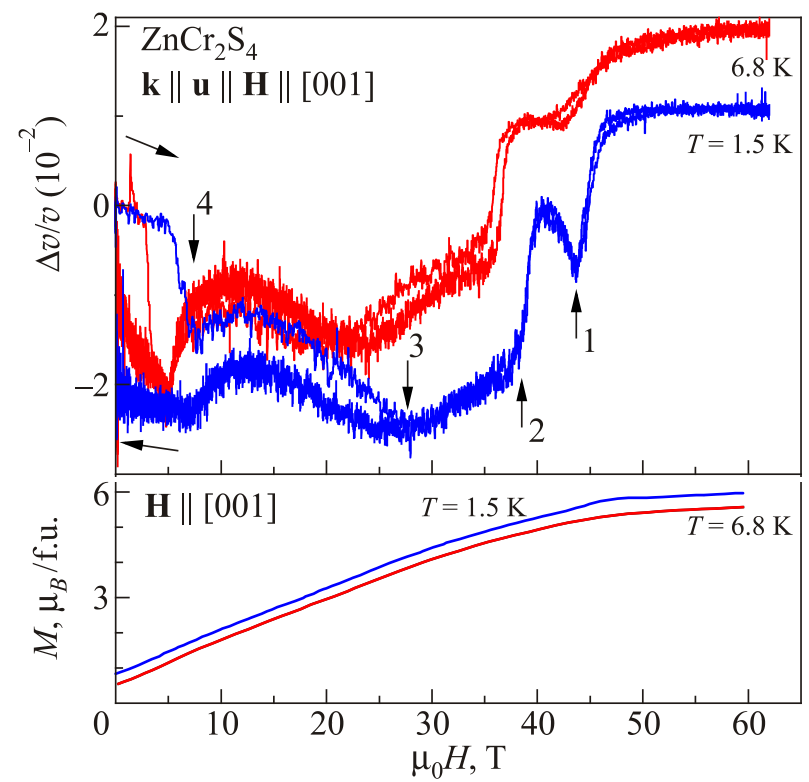

Fig. 13. (Color online) Relative change of the sound velocity, $\Delta v / v$, of the acoustic $c_{11}$ mode in $\mathrm{ZnCr}_{2} \mathrm{~S}_{4}$ at 1.5 and $6.8 \mathrm{~K}$ versus magnetic field applied along the [001] direction. Data for up and down field sweeps and sweep directions are shown. The characteristic anomalies in the sound velocity are numbered for the $1.5 \mathrm{~K}$ data. The lower panel shows corresponding magnetization curves. The magnetization curves are shifted along the vertical axis for clarity. See Ref. 38 for more details.

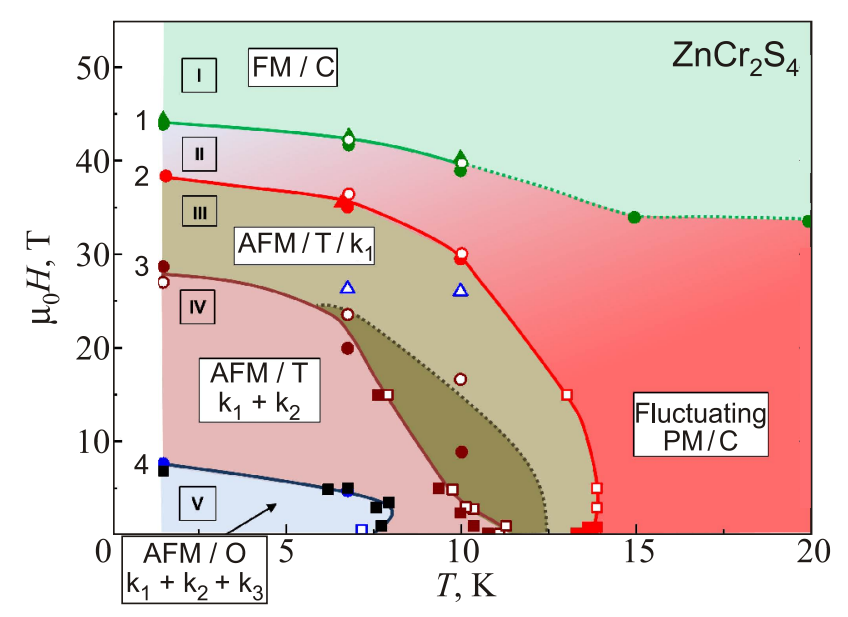

Fig. 14. (Color online) $H-T$ phase diagram of $\mathrm{ZnCr}_{2} \mathrm{~S}_{4}$ obtained from the ultrasound and magnetization measurements. Numbers in the low-temperature range correspond to sound-velocity anomalies marked in Fig. 13 for the $1.5 \mathrm{~K}$ data. For description of various phases see Ref. 38 .

antiferromagnetic ordering in $\mathrm{CdCr}_{2} \mathrm{O}_{4}$ at $T_{N}=7.8 \mathrm{~K}$ is accompanied by a cubic $(F d \overline{3} m)$ to tetragonal $\left(I 4_{1} /\right.$ amd $)$ structural change [55], which can be considered as a spin Jahn-Teller phase transition driven by spin frustrations [48]. Spin-orbit coupling is negligible in this compound.

Magnetization data exhibit a broad plateau of $1 / 2$ of the saturation magnetization between 28 and about $60 \mathrm{~T}$ [48-50]. The fully polarized state is achieved above $90 \mathrm{~T}$ in this system $[49,50]$. Only a minor anisotropy has been observed [48]. Pulsed-field ultrasound data show a drastic change in the sound velocity just below and above the $1 / 2$ magnetization plateau (Fig. 15). These results together with a large sound-velocity change in the vicinity of $T_{N}$ give evidence for a pronounced interplay between spin and lattice degrees of freedom in $\mathrm{CdCr}_{2} \mathrm{O}_{4}$ [35,51-54,57]. The sound velocity first decreases, then reveals a jump at the magne-

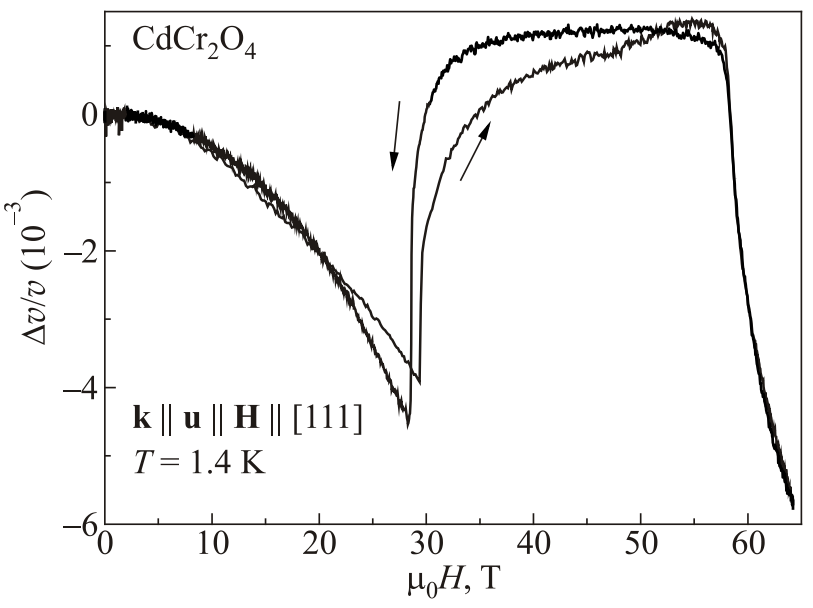

Fig. 15. Change of the sound velocity of the $c_{L}$ mode in $\mathrm{CdCr}_{2} \mathrm{O}_{4}$ measured in a pulsed magnetic field at $1.4 \mathrm{~K}$ and using an ultrasound frequency of $81 \mathrm{MHz}$. The experimental geometry is $\mathbf{H}\|\mathbf{k}\| \mathbf{u} \|[111]$. 
tic field where the magnetization plateau appears. This anomaly corresponds to a first-order phase transition from the spiral AF structure to a collinear spin configuration with three spins up and one spin down at each $\mathrm{Cr}^{3+}$ tetrahedron [48]. Previously, a large magnetostriction has been reported at this phase transition [48]. A cubic crystallographic structure has been suggested from high-field x-ray data in the plateau state [56]. Neutron-scattering experiments in pulsed magnetic fields showed that the magnetic structure in the plateau phase had a cubic $P 4_{3} 32$ symmetry [55]. It has been proposed that a lattice distortion stabilizes the 3-up 1-down collinear spin configuration [52,53]. Indeed, there is only a slight change of the sound velocity within the magnetization-plateau range. This is because the spin configuration is locked here [48].

The plateau terminates at approximately $58 \mathrm{~T}$, confirmed by a sharp anomaly, i.e., an abrupt decrease in the sound velocity. This anomaly corresponds to a phase transition to a non-collinear canted spin configuration $[50,53]$. It is interesting to note that the hysteresis in the sound velocity takes place not only at the first-order phase transition around $28 \mathrm{~T}$, but spreads along the whole plateau region up to $58 \mathrm{~T}$, showing a complicated interplay between the spin and lattice degrees of freedom.

The lattice distortion complicates the theoretical analysis of the acoustic properties. The sound-velocity change, which takes place between 58 and $63 \mathrm{~T}$, is even larger than the anomaly at $28 \mathrm{~T}$. The highest applied magnetic field of $63 \mathrm{~T}$ is not sufficient to detect the complete sound-velocity change. No hysteresis has been detected at $58 \mathrm{~T}$, pointing to a second-order phase transition. It is worth noting that the magnetization exhibits only a smooth, kink-like anomaly at this phase transition [49,50]. A transverse spin order which is equivalent to a Bose-Einstein condensation of magnons is predicted from quantum-fluctuation theory at magnetic fields just above the plateau [57]. Detailed theoretical consideration of the spin-phonon coupling in $\mathrm{CdCr}_{2} \mathrm{O}_{4}$ and its effect on the sound velocity and sound attenuation is given in Ref. 35 .

\subsection{Uranium-based antiferromagnets}

We now turn to another class of antiferromagnets, uranium-intermetallic compounds. These materials are subject of intensive fundamental research because of the special character of the $5 f$ states. Interesting phenomena (e.g., heavy fermions, non-conventional superconductivity, nonFermi-liquid behavior) have been discovered in uraniumintermetallics [58-60] The magneto-elastic and electronic properties of uranium intermetallics are related to the degree of itinerancy of the $5 f$ electrons. The hybridization of the uranium $5 f$ states with the valence-electron states plays a major role in the formation of $5 f$ magnetic moments [58]. Magnetically ordered uranium compounds typically have a huge magnetic anisotropy. Their study requires high-quality single crystals and high magnetic fields. Here, we review results for two uranium compounds, $\mathrm{UCo}_{2} \mathrm{Si}_{2}$ and $\mathrm{UCu}_{0.95} \mathrm{Ge}$. We show that in these materials magneto-elastic interactions play an important role, too.

$\mathrm{UCo}_{2} \mathrm{Si}_{2}$ has a tetragonal crystal structure (space group $I 4 / \mathrm{mmm}$ ) and orders antiferromagnetically at $T_{N} \approx 83 \mathrm{~K}$. The magnetic structure obtained from powder neutron diffraction consists of ferromagnetic basal-plane layers with $\mathrm{U}$ magnetic moments of $1.42 \mu_{B}$ (at $4.2 \mathrm{~K}$ ) oriented parallel to the $c$ axis. The layers are coupled in the simple sequence "+ - + -" (AFM type-I structure) [64]. The magnetic moment is localized only on the $U$ atoms.

Figure 16 shows the temperature dependence of the specific heat divided by temperature, as well as the sound velocity and sound attenuation of the acoustic $c_{11}$ mode measured in $\mathrm{UCo}_{2} \mathrm{Si}_{2}$ at zero magnetic field. Wellpronounced anomalies are seen in all quantities at the AFM ordering. The sound-velocity change, $\Delta v / v$, shows a deep minimum of $1.2 \%$ reflecting a pronounced softening of the lattice, whereas the sound attenuation, $\Delta \alpha$, exhibits a sharp peak of $6 \mathrm{~dB} / \mathrm{cm}$, apparently due to the spin-lattice relaxation effects in the vicinity of the transition [68]. The observed features prove a strong magneto-elastic coupling in $\mathrm{UCo}_{2} \mathrm{Si}_{2}$.

In a magnetic field of $45 \mathrm{~T}(T=1.5 \mathrm{~K})$ applied along the $c$ axis a metamagnetic transition to the ferrimagnetic

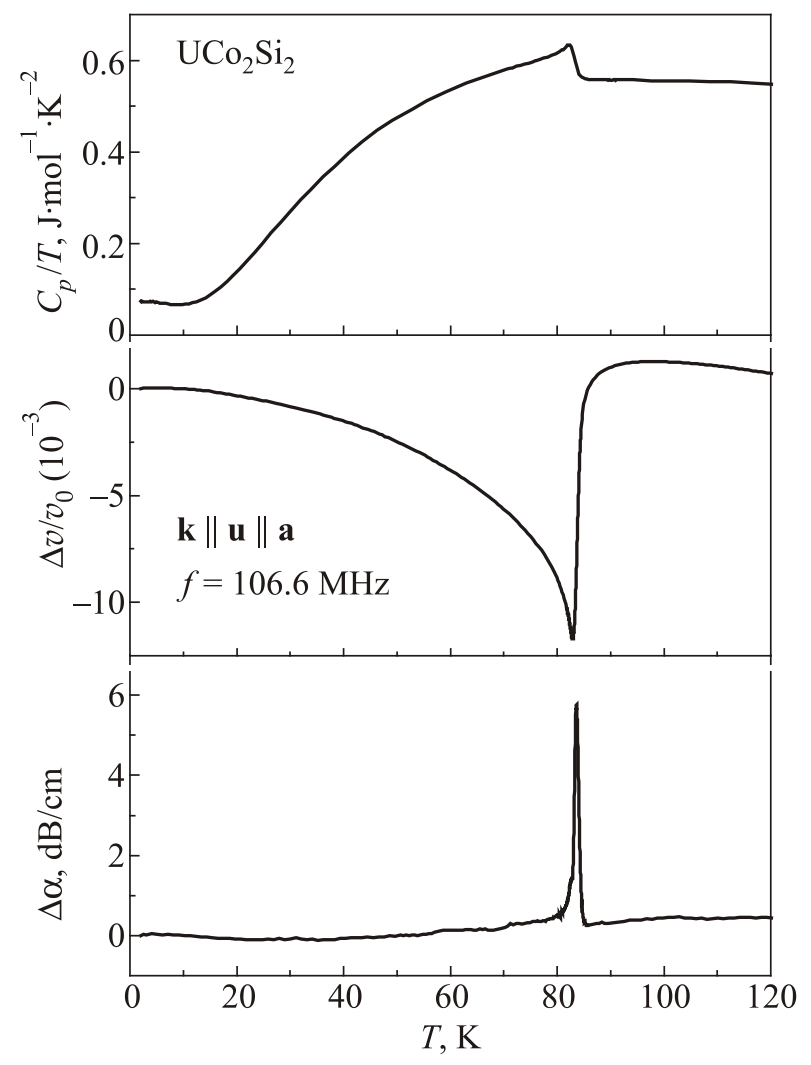

Fig. 16. Temperature dependence of the specific heat divided by temperature, $C_{p} / T$, the relative change of the sound velocity, $\Delta v / v$, and of the sound attenuation, $\Delta \alpha$, for the acoustic $c_{11}$ mode $(\mathbf{k}\|\mathbf{u}\| \mathbf{a})$ in $\mathrm{UCo}_{2} \mathrm{Si}_{2}$ measured at zero magnetic field. 
state having " ++ +" configuration has been observed in the magnetization as well as in the sound velocity and sound attenuation (Fig. 17). The transition is very sharp and exhibits a small but non-negligible hysteresis of $0.16 \mathrm{~T}$. With increasing temperature, it becomes broader and vanishes at $T_{N}$ [61]. The magnetization measured along the hard $a$ axis shows no transition and is linear up to the highest fields applied in the experiment.

A broad minimum at about $30 \mathrm{~T}$ is seen in $\Delta v / v$ and a sharp $0.6 \%$ step-like increase at $45 \mathrm{~T}$ is observed at the metamagnetic transition, accompanied by a very sharp peak in the sound attenuation. The origin of the lattice softening at about $30 \mathrm{~T}$ is unclear, no features are seen in the magnetization at this magnetic field. The $H-T$ phase diagram of $\mathrm{UCo}_{2} \mathrm{Si}_{2}$ was obtained from these experiments [61]. The exchange-striction approach based on the Eqs. (1)-(3) and taking into account the magnetic susceptibility of the $\mathrm{UCo}_{2} \mathrm{Si}_{2}$ spin subsystem can sucsessfully explain the main features in the acoustic properties [61].

Another interesting uranium-based antiferromagnet $\left(T_{N}=\right.$ $=48 \mathrm{~K}$ ) is $\mathrm{UCu}_{0.95} \mathrm{Ge}$ which crystallizes in the hexagonal structure. According to a neutron-diffraction study on polycrystalline samples [69], the magnetic unit cell contains four $\mathrm{U}$ ions, and the $\mathrm{U}$ magnetic moments lie in the basal $a b$ plane. In the $z=0$ layer, they form an angle of $\pi / 3$ with the $a$ axis. Within the layer, the $\mathrm{U}$ moments form ferromagnetic chains along the $a$ axis, which are coupled

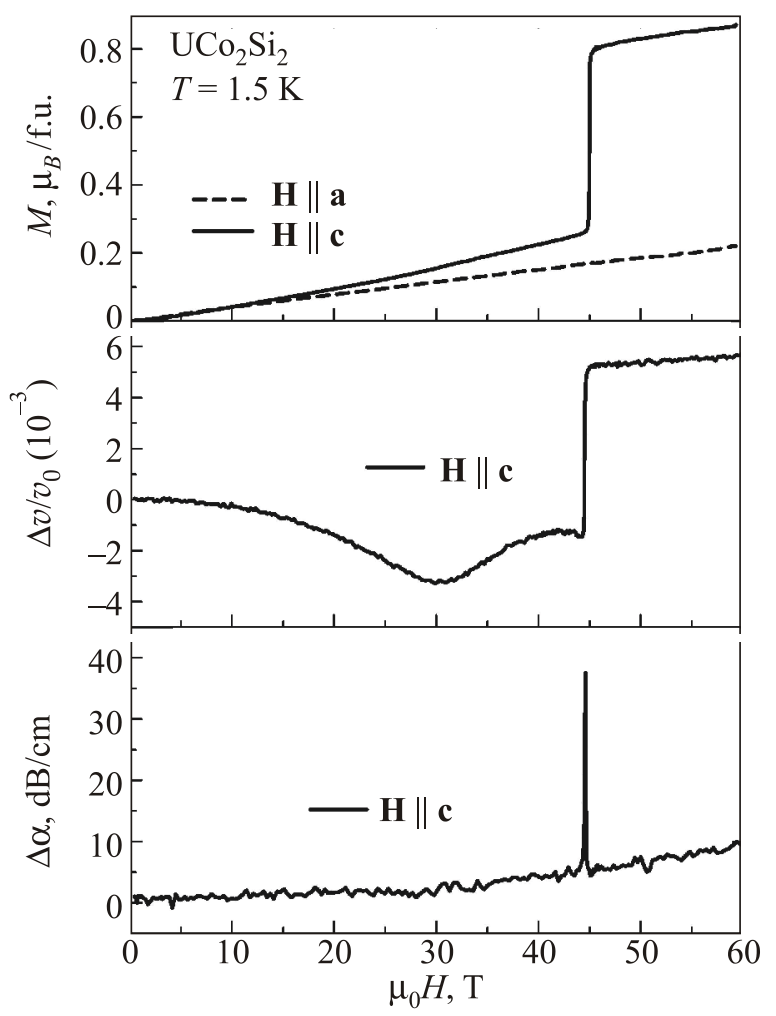

Fig. 17. Field dependence of the magnetization, $M$, the relative change of the sound velocity, $\Delta v / v$, and of the sound attenuation, $\Delta \alpha$, for the acoustic $c_{11}$ mode $(\mathbf{k}\|\mathbf{u}\| \mathbf{a})$ in $\mathrm{UCo}_{2} \mathrm{Si}_{2}$ measured at $1.5 \mathrm{~K}$. antiferromagnetically. In the neighboring layer at $z=1 / 2$, the moments are rotated by $\pi / 2$ with respect to the layer at $z=0$. The $\mathrm{U}$ magnetic moment $\mu_{\mathrm{U}}$ is equal to $2.0 \mu_{B}$ [69]. However, this value might be overestimated.

Figure 18 shows temperature dependence of the specific heat, as well as changes of the sound velocity and of the sound attenuation for the acoustic modes $c_{11}$ and $c_{33}$ modes measured in a $\mathrm{UCu}_{0.95} \mathrm{Ge}$ single crystal. At $T_{N}$, $\Delta v / v$ exhibits a sharp softening for both propagation directions, whereas $\Delta \alpha$ displays a pronounced anisotropy with rather small effect for the acoustic $c_{33}$ mode.

Figure 19 shows the field dependence of the sound velocity and attenuation, measured at $4.2 \mathrm{~K}$, for longitudinal waves propagating along the $a$ and $c$ axes with the magnetic field applied along the same axes. The field dependent magnetization for the two field orientations is shown as well in the upper panel of Fig. 19. For fields applied along the $a$ axis we found a first-order phase transition in $\mathrm{UCu}_{0.95} \mathrm{Ge}$ at $61 \mathrm{~T}(T=4.2 \mathrm{~K})$ [62]. For field applied along the $c$ axis, a first-order phase transition occurs at the much lower magnetic field of $38 \mathrm{~T}$ [66]. In both directions, the magnetization tends to saturate at $1.35 \mu_{B}$ per formula unit in the fully polarized state. The field-induced transitions as well as the AFM ordering are accompanied by pronounced anomalies in the sound velocity and sound

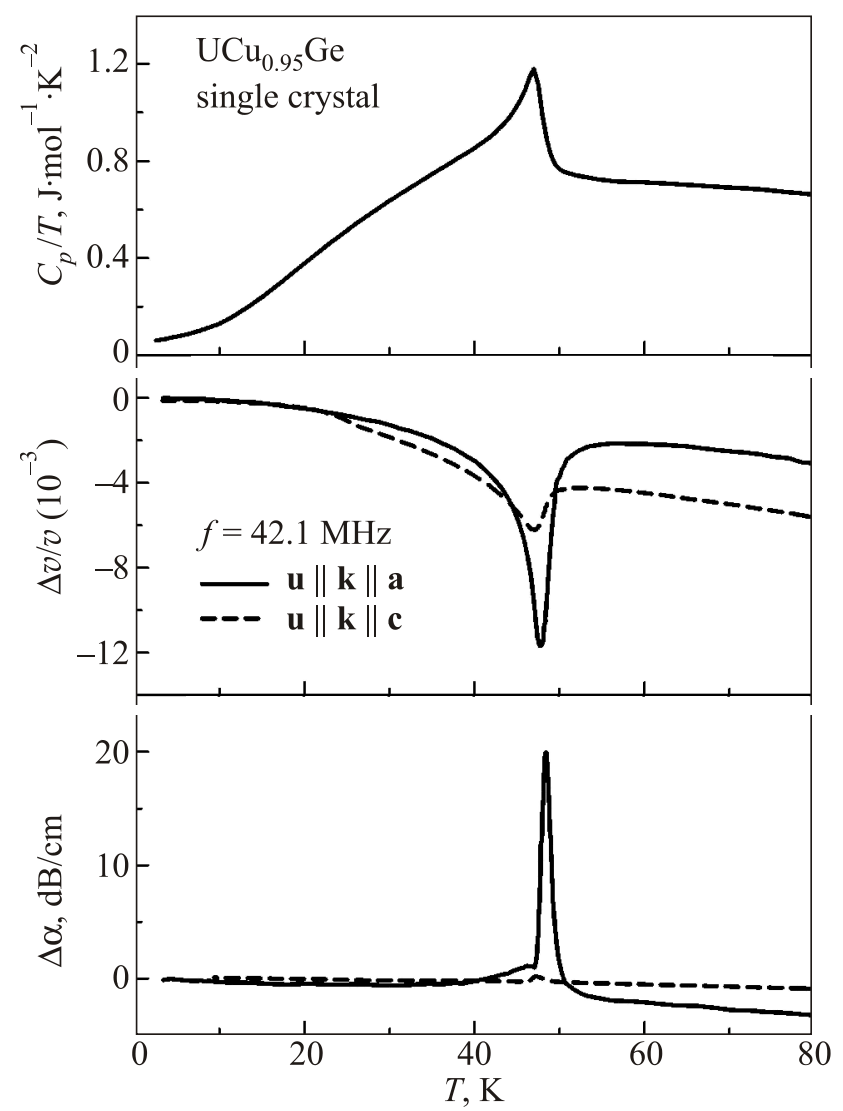

Fig. 18. Temperature dependence of the specific heat divided by temperature, $C_{p} / T$, the relative change of the sound velocity, $\Delta v / v$, and of the sound attenuation, $\Delta \alpha$, for the acoustic modes $c_{11}$ and $c_{33}$ in $\mathrm{UCu}_{0.95} \mathrm{Ge}$ measured at zero magnetic field. 


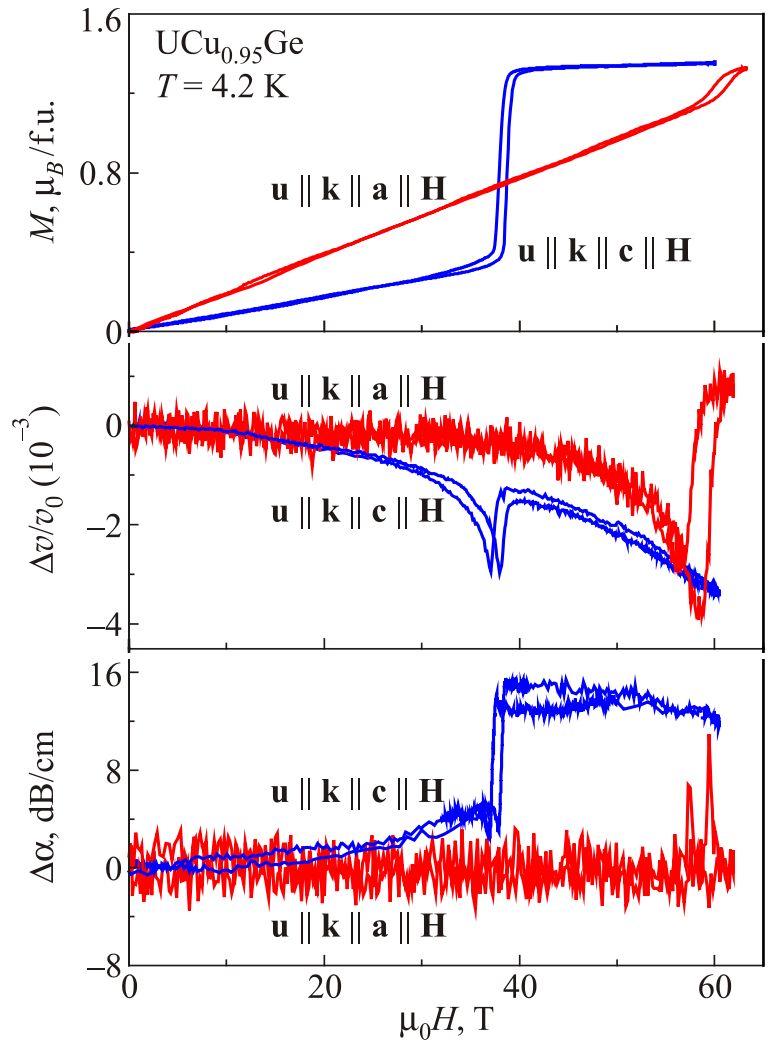

Fig. 19. (Color online) Field dependence of the magnetization, $M$, the relative change of the sound velocity, $\Delta v / v$, and of the sound attenuation, $\Delta \alpha$, for the acoustic modes $c_{11}$ and $c_{33}$ in $\mathrm{UCu}_{0.95} \mathrm{Ge}$ measured in pulsed magnetic fields applied along the $a$ and $c$ directions at $4.2 \mathrm{~K}$.

attenuation. At the critical field, the sound velocity behaves qualitatively in a comparable way for both field directions. Quantitatively, the minimum in $\Delta v / v$ is deeper for the field aligned along $a$ direction, whereas the magnetization jump for fields along the $c$ axis is much larger. In the sound attenuation a sharp maximum in $\Delta \alpha$ is observed at the critical field for $\mathbf{H} \| \mathbf{a}$, whereas a step appears for the field applied along the $c$ axis. Apparently the magneto-elastic coupling differs in both cases. The mean-field approximation provides a good basis for a qualitatively description of the observed behavior of the magnetic and magneto-acoustic properties of $\mathrm{UCu}_{0.95} \mathrm{Ge}$ and deliver qualitative agreement with the experimental data [62].

\section{Conclusions}

In this short review we have given selected examples of spin-lattice effects in antiferromagnetic materials, namely low-dimensional spin systems, frustrated chromium spinels, and uranium-based compounds. We demonstrated that the ultrasound technique combined with low temperatures and very high magnetic fields is a proper tool to investigate spinlattice effects.

Spin-strain interactions play important role in all materials studied. In the low-dimensional spin systems quantum fluctuations couple to the lattice leading to renormaliza- tions of the sound velocity and sound attenuation in the vicinity of quantum critical points. In the frustrated chromium spinels spin-strain coupling is crucial and determines the underlying physics of these compounds. In these materials external magnetic fields change the balance between the competing exchange interactions leading to fascinating magneto-structural states and some unusual islands of metastable states in the $H-T$ phase diagram. In the uraniumbased compounds magnetoelastic interactions renormalize acoustic properties in the vicinity of the spontaneous and field-induced magnetic transitions. It is worth to note that the ultrasound characteristics are very sensitive to phase transitions allowing to map out phase diagrams. Furthermore, our studies show that in many cases the models describing antiferromagnetic materials should explicitly include the lattice degrees of freedom and spin-phonon interactions in order to address correctly their physical properties.

We thank Y. Skourski, V. Felea, A. Sytcheva, and O. Chiatti for their valuable contributions in the course of these studies. We acknowledge the support of HLD at HZDR, member of the European Magnetic Field Laboratory (EMFL). The research has been partly supported by the DFG via TRR 80 (Augsburg-Münich), by the Chech Science Foundation (grant No. P204/12/0150), by the Czech Academy of Sciences (grant M100101203), and within the program of Czech Research Infrastructure (Project No. LM2011025). A.A.Z. acknowledges the support from the Institute for Chemistry of V.N. Karasin Kharkov National University.

1. B. Lüthi, Physical Acoustics in the Solid State, Springer, Berlin (2005).

2. Physical Acoustics, Vols. I-XXV, Academic Press, New York and London (1965-1999).

3. R. Truell, C. Elbaum, and B.B Chick, Ultrasonic Methods in Solid State Physics, Academic Press, New York and London (1969).

4. J.W. Tucker and V.W. Rampton, Microwave Ultrasonics in Solid State Physics, North-Holland Publishing Company, Amsterdam (1972).

5. A.K. Zvezdin, V.M. Matveev, A.A. Mukhin, and A.I. Popov, Redkozemel'nie Ioni v Magneto-uporyadochennih kristallah, Nauka, Moscow (1985).

6. M.E. Lines, Phys. Rep. 55, 133 (1979).

7. I.K. Kamilov and Kh. Aliev, Phys. Usp. 41, 865 (1998).

8. G.A. Gehring and K.A. Gehring, Rep. Prog. Phys. 38, 1 (1975).

9. E.A. Turov and V.G. Shavrov, Sov. Phys. Usp. 26, 593 (1983).

10. B. Wolf, B. Lüthi, S. Schmidt, H. Schwenk, M. Sieling, S. Zherlitsyn, and I. Kouroudis, Physica B 294-295, 612 (2001).

11. S. Zherlitsyn, B. Wustmann, T. Herrmannsdörfer, and J. Wosnitza, J. Low Temp. Phys. 170, 447 (2013). 
12. S. Zherlitsyn, B. Wustmann, T. Herrmannsdörfer, and J. Wosnitza, IEEE Trans. Appl. Superconduct. 22, 4300603 (2012).

13. O. Chiatti, A. Sytcheva, J. Wosnitza, S. Zherlitsyn, A.A. Zvyagin, V.S. Zapf, M. Jaime, and A. Paduan-Filho, Phys. Rev. B 78, 094406 (2008).

14. O. Chiatti, S. Zherlitsyn, A. Sytcheva, J. Wosnitza, A.A. Zvyagin, V.S. Zapf, M. Jaime, and A. Paduan-Filho, J. Phys.: Conf. Series 150, 042016 (2009).

15. S. Zherlitsyn, O. Chiatti, A. Sytcheva, J. Wosnitza, A.A. Zvyagin, V.S. Zapf, M. Jaime, and A. Paduan-Filho, J. Phys.: Conf. Series 145, 012069 (2009).

16. A. Sytcheva, O. Chiatti, J. Wosnitza, S. Zherlitsyn, A.A. Zvyagin, R. Coldea, and Z. Tylczynski, Phys. Rev. B 80, 224414 (2009).

17. A. Sytcheva, O. Chiatti, J. Wosnitza, S. Zherlitsyn, A.A. Zvyagin, R. Coldea, and Z. Tylczynski, J. Low Temp. Phys. 159, 109 (2010).

18. V.S. Zapf, D. Zocco, B.R. Hansen, M. Jaime, N. Harrison, C.D. Batista, M. Kenzelmann, C. Niedermayer, A. Lacerda, and A. Paduan-Filho, Phys. Rev. Lett. 96, 077204 (2006).

19. T. Radu, H. Wilhelm, V. Yushankhai, D. Kovrizhin, R. Coldea, Z. Tylczynski, T. Lühmann, and F. Steglich, Phys. Rev. Lett. 95, 127202 (2005).

20. S.E. Sebastian, V.S. Zapf, N. Harrison, C.D. Batista, P.A. Sharma, M. Jaime, I.R. Fisher, and A. Lacerda, Phys. Rev. Lett. 96, 189703 (2006).

21. T. Radu, H. Wilhelm, V. Yushankhai, D. Kovrizhin, R. Coldea, Z. Tylczynski, T. Lühmann, and F. Steglich, Phys. Rev. Lett. 96, 189704 (2006)

22. F. Weickert, R. Küchler, A. Steppke, L. Pedrero, M. Nicklas, M. Brando, F. Steglich, M. Jaime, V.S. Zapf, A. PaduanFilho, K.A. Al-Hassanieh, C.D. Batista, and P. Sengupta, Phys. Rev. B 85, 184408 (2012).

23. S.A. Zvyagin, J. Wosnitza, C.D. Batista, M. Tsukamoto, N. Kawashima, J. Krzystek, V.S. Zapf, M. Jaime, N.F. Oliveira, Jr., and A. Paduan-Filho, Phys. Rev. Lett. 98, 047205 (2007).

24. A. Paduan-Filho, X. Gratens, and N.F. Oliveira, Jr., Phys. Rev. B 69, 020405R (2004).

25. V.S. Zapf, V.F. Correa, P. Sengupta, C.D. Batista, M. Tsukamoto, N. Kawashima, P. Egan, C. Pantea, A. Migliori, J.B. Betts, M. Jaime, and A. Paduan-Filho, Phys. Rev. B 77, 020404R (2008).

26. M. Tachiki and S. Maekawa, Progr. Theor. Phys. 51, 1 (1974).

27. See, e.g., A.A. Zvyagin, Finite Size Effects in Correlated Electron Models: Exact Results, Imperial College Press, London (2005).

28. F. Alet, A. Walczak, and M. Fisher, Physica A 369, 122 (2006).

29. R. Coldea, D.A. Tennant, and Z. Tylczynski, Phys. Rev. B 68, 134424 (2003).

30. R. Coldea, D.A. Tennant, A.M. Tsvelik, and Z. Tylczynski, Phys. Rev. Lett. 86, 1335 (2001).

31. Y. Tokiwa, T. Radu, R. Coldea, H. Wilhelm, Z. Tylczynski, and F. Steglich, Phys. Rev. B 73, 134414 (2006).

32. R. Coldea, D.A. Tennant, K. Habicht, P. Smeibidl, C. Wolters, and Z. Tylczynski, Phys. Rev. Lett. 88, 137203 (2002).
33. A. Kreisel, P. Kopietz, P.T. Cong, B. Wolf, and M. Lang, Phys. Rev. B 84, 024414 (2011).

34. T. Rudolf, C. Kant, F. Mayr, J. Hemberger, V. Tsurkan, and A. Loidl, New J. Phys. 9, 76 (2007).

35. S. Bhattacharjee, S. Zherlitsyn, O. Chiatti, A. Sytcheva, J. Wosnitza, R. Moessner, M.E. Zhitomirsky, P. Lemmens, V. Tsurkan, and A. Loidl, Phys. Rev. B 83, 184421 (2011).

36. S. Zherlitsyn, O. Chiatti, A. Sytcheva, J. Wosnitza, S. Bhattacharjee, R. Moessner, M.E. Zhitomirsky, P. Lemmens, V. Tsurkan, and A. Loidl, J. Low Temp. Phys. 159, 134 (2010).

37. V. Tsurkan, S. Zherlitsyn, S. Yasin, V. Felea, Y. Skourski, J. Deisenhofer, H.-A. Krug von Nidda, J. Wosnitza, and A. Loidl, Phys. Rev. Lett. 110, 115502 (2013).

38. V. Tsurkan, S. Zherlitsyn, V. Felea, S. Yasin, Yu. Skourski, J. Deisenhofer, H.-A. Krug von Nidda, P. Lemmens, J. Wosnitza, and A. Loidl, Phys. Rev. Lett. 106, 247202 (2011).

39. V. Felea, S. Yasin, A. Günther, J. Deisenhofer, H.-A. Krug von Nidda, S. Zherlitsyn, V. Tsurkan, P. Lemmens, J. Wosnitza, and A. Loidl, Phys. Rev. B 86, 104420 (2012).

40. Y. Kino and B. Lüthi, Solid State Commun. 9, 805 (1971).

41. N. Menyuk, K. Dwight, and A. Wold, J. Phys. France 25, 528 (1964).

42. K. Tomiyasu, J. Fukunaga, and H. Suzuki, Phys. Rev. B 70, 214434 (2004).

43. L.J. Chang, D.J. Huang, W.-H. Li, S.-W. Cheong, W. Ratcliff, and J.W. Lynn, J. Phys.: Condens. Matter 21, 456008 (2009).

44. A.V. Pronin, M. Uhlarz, R. Beyer, T. Fischer, J. Wosnitza, B.P. Gorshunov, G.A. Komandin, A.S. Prokhorov, M. Dressel, A.A. Bush, and V.I. Torgashev, Phys. Rev. B 85, 012101 (2012).

45. D. Kamenskyi, H. Engelkamp, T. Fischer, M. Uhlarz, J. Wosnitza, B.P. Gorshunov, G.A. Komandin, A.S. Prokhorov, M. Dressel, A.A. Bush, V.I. Torgashev, and A.V. Pronin, Phys. Rev. B 87, 134423 (2013).

46. Y. Yamasaki, S. Miyasaka, Y. Kaneko, J.-P. He, T. Arima, and Y. Tokura, Phys. Rev. Lett. 96, 207204 (2006).

47. T. Arima, Y. Yamasaki, T. Goto, S. Iguchi, K. Ohgushi, S. Miyasaka, and Y. Tokura, J. Phys. Soc. Jpn. 76, 023602 (2007).

48. H. Ueda, H.A. Katori, H. Mitamura, T. Goto, and H. Takagi, Phys. Rev. Lett. 94, 047202 (2005).

49. A. Miyata, S. Takeyama, and H. Ueda, Phys. Rev. B 87, 214424 (2013).

50. E. Kojima, A. Miyata, S. Miyabe, S. Takeyama, H. Ueda, and Y. Ueda, Phys. Rev. B 77, 212408 (2008).

51. J.-H. Chung, M. Matsuda, S.-H. Lee, K. Kakurai, H. Ueda, T.J. Sato, H. Takagi, K.-P. Hong, and S. Park, Phys. Rev. Lett. 95, 247204 (2005)

52. M. Matsuda, M. Takeda, M. Nakamura, K. Kakurai, A. Oosawa, E. Lelièvre-Berna, J.-H. Chung, H. Ueda, H. Takagi, and S.-H. Lee, Phys. Rev. B 75, 104415 (2007).

53. K. Penc, N. Shannon, and H. Shiba, Phys. Rev. Lett. 93, 197203 (2004).

54. O. Tchernyshyov, R. Moessner, and S.L. Sondhi, Phys. Rev. $B$ 66, 064403 (2002). 
55. M. Matsuda, K. Ohoyama, S. Yoshii, H. Nojiri, P. Frings, F. Duc, B. Vignolle, G.L. J.A. Rikken, L.-P. Regnault, S.-H. Lee, H. Ueda, and Y. Ueda, Phys. Rev. Lett. 104, 047201 (2010).

56. T. Inami, K. Ohwada, M. Tsubota, Y. Murata, Y.H. Matsuda, H. Nojiri, H. Ueda, and Y. Murakami, J. Phys. 51, 502 (2006).

57. D.L. Bergman, R. Shindou, G.A. Fiete, and L. Balents, Phys. Rev. Lett. 96, 097207 (2006).

58. V. Sechovsky and L. Havela, in: Handbook of Magnetic Materials, K.H.J. Buschow (ed.), vol. 11, Amsterdam, North Holland (1998), and references therein.

59. A. Szytula and J. Leciejewicz, in: Handbook on the Physics and Chemistry of the Rare Earths, K.A. Gschneider, Jr., and L. Eyring (eds.), Elsevier Science B.V., Amsterdam (1989), Vol. 12, p. 133, and references therein.

60. J.A. Mydosh and P.M. Oppeneer, Rev. Mod. Phys. 83, 1301 (2011).

61. A.V. Andreev, S. Yasin, Y. Skourski, A.A. Zvyagin, S. Zherlitsyn, and J. Wosnitza, Phys. Rev. B 87, 214409 (2013).
62. A.A. Zvyagin, S. Yasin, Y. Skourski, A.V. Andreev, S. Zherlitsyn, and J. Wosnitza, J. Alloys Compd. 528, 51 (2012).

63. A.V. Andreev, Y. Skourski, S. Yasin, S. Zherlitsyn, and J. Wosnitza, J. Magn. Magn. Mater. 324, 3413 (2012).

64. L. Chelmicki, J. Leciejewicz, and A. Zygmunt, J. Phys. Chem. Solids 46, 529 (1985).

65. A.V. Andreev, Y. Skourski, M.D. Kuz'min, S. Yasin, S. Zherlitsyn, R. Daou, J. Wosnitza, A. Iwasa, A. Kondo, A. Matsuo, and K. Kindo, Phys. Rev. B 83, 184422 (2011).

66. S. Yasin, A.V. Andreev, Y. Skourski, J. Wosnitza, S. Zherlitsyn, and A.A. Zvyagin, Phys. Rev. B 83, 134401 (2011).

67. S. Yasin, A.V. Andreev, A. Sytcheva, J. Wosnitza, and S. Zherlitsyn, J. Low Temp. Phys. 159, 105 (2010).

68. K. Kawasaki, Phys. Lett. A 29, 406 (1969).

69. J. Leciejewicz, A. Szytula, and A. Zygmunt, J. Magn. Magn. Mater. 97, 219 (1991). 